\title{
Dípteros fanídeos vetores de ovos de Dermatobia hominis em Campo Grande, Mato Grosso do Sul ${ }^{1}$
}

\author{
Patrícia R. Gomes ${ }^{2}$, Wilson W. Koller ${ }^{3}$, Alberto Gomes ${ }^{3}$, Cláudio J. B. Carvalho ${ }^{4}$ e José \\ R. Zorzatto ${ }^{5}$
}

\begin{abstract}
Gomes P.R., Koller W.W., Gomes A., Carvalho C.J.B. \& Zorzatto J.R. 2002. [Fanniid diptera vectors of Dermatobia hominis eggs in Campo Grande, MS, Brazil.] Dípteros fanídeos vetores de ovos de Dermatobia hominis em Campo Grande, Mato Grosso do Sul. Pesquisa Veterinária Brasileira 22 (3): 114-118. Laboratório de Ectoparasitologia, Embrapa Gado de Corte, BR 262 Km 4, Cx. Postal 154, Campo Grande, MS 79002-970, Brazil.

This study was undertaken to determine the epidemiological importance of fanniid flies on warble fly infestations. The local species of Fanniidae as well as those utilized by Dermatobia hominis to carry its eggs were identified, and the population dynamics of the most abundant species were studied. In order to accomplish this task, five traps with rotten bovine liver as bait were used. These traps were settled into a ciliate forest bordered by pastures and grazed continuously by cattle. The experiment was carried out at Embrapa Gado de Corte, in Campo Grande, Mato Grosso do Sul, Brazil, situated at $20^{\circ} 27^{\prime}$ S and $54^{\circ} 37^{\prime} \mathrm{W}$. The capture of the insects was done weekly, from August 9, 1999 to August 3, 2000. A total of 40,629 fanniid flies which belonged to five different species of the genera Fannia were captured: F. pusio, F. heydenii, $F$. bahiensis and $F$. longipila, and one species which needs further identification. The most frequent species was $F$. pusio, with $63.20 \%$ of the total captured flies, followed by $F$. heydenii with $28.82 \%$. Eggs of $D$. hominis were observed only on $0.44 \%$ (45 specimens) of the $F$. heydenii females captured, mainly on the ones captured in August and September. The average number for $D$. hominis eggs, per specimen, was $15.98 \pm 7.13$. The only vector body region, where the eggs of $D$. hominis were found, was the abdomen. $F$. heydenii predominated during the dry season (May to September) and at the beginning of the rainy season (October and November). The number of specimens bearing eggs of $D$. hominis was greater at the end of the annual dry season, what explains the high incidence of this parasite in cattle during September and October.
\end{abstract}

INDEX TERMS: Vectors, warble fly, epidemiology, Brazilian savannas.

RESUMO.- Determinou-se a importância epidemiológica de dípteros Fanniidae na infestação de mosca-do-berne, por meio da identificação das espécies presentes, da determinação daquelas utilizadas por Dermatobia hominis na veiculação de

\footnotetext{
${ }^{1}$ Aceito para publicação em 10 de agosto de 2002.

Parte da Dissertação de Mestrado apresentada ao Departamento de Medicina Veterinária, Curso de Biologia Parasitária, Convênio FIOCRUZ/UFMS.

2Pós-Graduando (Mestrado) do Curso de Biologia Parasitária, Departamento de Medicina Veterinária, Convênio FIOCRUZ/UFMS.

${ }^{3}$ Embrapa Gado de Corte, Rodovia BR 262 Km 4, Cx. Postal 154, Campo Grande, MS 79002-970. E-mail: koller@cnpgc.embrapa.br

${ }^{4}$ Departamento de Zoologia, Universidade Federal do Paraná.

${ }^{5}$ Departamento de Computação e Estatística, Universidade Federal de Mato Grosso do Sul (UFMS).
}

seus ovos, bem como, pelo conhecimento da dinâmica populacional das espécies mais abundantes. Foram utilizadas cinco armadilhas iscadas com fígado bovino cru deteriorado e colocadas em uma mata ciliar margeada por uma área de pastagem com presença constante de bovinos. $O$ estudo foi desenvolvido em uma área da Embrapa Gado de Corte, em Campo Grande, Estado de Mato Grosso do Sul, Brasil, localizada a $20^{\circ} 27^{\prime} \mathrm{S}$ e $54^{\circ} 37^{\prime} \mathrm{W}$. A captura dos insetos foi realizada semanalmente durante o período de 09/08/1999 a 03/ 08/2000. Foi capturado um total de 40.629 moscas da família Fanniidae, pertencendo a cinco espécies do gênero Fannia: $F$. pusio, $F$. heydenii, $F$. bahiensis e $F$. longipila, e uma a ser identificada. A espécie mais freqüente foi $F$. pusio, com $63,20 \%$ do total capturado, seguida de $F$. heydenii, com $28,82 \%$. Somente $0,44 \%$ do total de fêmeas de $F$. heydenii (45 exemplares) 
capturadas, principalmente nos meses de agosto e setembro, portavam ovos de $D$. hominis e o número médio, por indivíduo, foi de $15,98 \pm 7,13$. Observaram-se ovos de $D$. hominis apenas na região abdominal dos vetores. F. heydenii predominou no período seco (maio a setembro) e início do período chuvoso do ano (outubro e novembro). $O$ número de exemplares portando ovos de $D$. hominis foi maior no final do período seco do ano, o que explica a alta incidência deste parasito em bovinos nos meses de setembro e outubro.

TERMOS DE INDEXAÇÃO: Vetores, mosca-do-berne, epidemiologia, Cerrado.

Classificação CNPq: 5.05.02.00-0 Medicina Veterinária Preventiva.

\section{INTRODUÇÃO}

Mateus (1967) relata que Dermatobia hominis, em seu estágio de larva, popularmente conhecida por "berne", constitui-se em um dos principais ectoparasitos de bovinos na América Latina, ocorrendo, segundo Roncalli (1984), desde o Sul do México até o Norte da Argentina, com exceção de El Salvador (por apresentar um solo seco) e do Chile (devido a condições climáticas desfavoráveis). Este último autor relata, ainda, que as regiões com temperaturas amenas e com alta umidade são favoráveis à presença da mosca, e que, tanto $D$. hominis quanto os seus vetores têm sido observados em regiões desde o nível do mar até a altitude de 1.600 metros. Este inseto, em seu estágio adulto, segundo Schonhorst (1988), entre outros, é primariamente habitante de florestas e as formações abertas parecem atuar como barreira à sua dispersão.

As larvas de $D$. hominis permanecem sobre bovinos por um período de 40 a 60 dias (Jobsen $\&$ Mourier 1972). Os principais prejuízos econômicos que o berne causa em bovinos são diminuição na produção de leite e carne, crescimento retardado, pré-disposição a enfermidades diversas e danos parciais ou totais no couro. $O$ berne nos animais causa a chamada míase furuncular ou dermatobiose, que se caracteriza pela formação de nódulos subcutâneos no hospedeiro, com a presença de uma ou mais larvas no interior (Gomes et al. 1998). Ocasionalmente, podem ocorrer infiltrações bacterianas, entre as quais as mais importantes são Staphylococcus aureus, S. epidermidis, S. warneri, Bacillus subtilis e Escherichia coli (Sancho et al. 1996). Os danos ao couro ainda acarretam prejuízos às indústrias do calçado e de produtos afins. As peles bovinas lesadas têm o seu valor comercial reduzido, pois peles com 10 a 20 perfurações disseminadas na região antero-dorsal perdem entre $30 \%$ e $40 \%$ do seu valor (Mateus 1979). Grisi et al. (2002) estimam que a pecuária nacional sofre uma perda anual da ordem de $\mathbf{2 5 0}$ milhões de dólares pela ação do berne.

Este parasito utiliza-se de organismos veiculadores de seus ovos, principalmente, dípteros. Bates (1943), estudando o comportamento de postura de $D$. hominis, na Colômbia, estabeleceu quatro características que os dípteros vetores dos ovos de $D$. hominis devem ter: a) hábito zoófilo; b) período de vôo diurno; c) tamanho moderado; d) atividade moderada.

Alguns trabalhos reportam as espécies vetoras, no Brasil, até agora incriminadas no ciclo de desenvolvimento de $D$. hominis, entre os quais destacam-se os de Oliveira (1986), Maia \& Gomes (1988), Paloschi et al. (1991), Brum et al. (1996), Gomes et al. (1998) e Rodríguez (1998). A lista mais recente de vetores de ovos de $D$. hominis observados no Brasil foi publicada por Guimarães \& Papavero (1999).

Na região de Cerrados de Mato Grosso do Sul, foi observado que $61,5 \%$ dos insetos capturados veiculando ovos de D. hominis pertenciam ao gênero Fannia, sendo que não houve determinação das espécies envolvidas (Gomes et al. 1998). Vários trabalhos relacionados à ocorrência de vetores de ovos de $D$. hominis têm registrado a importância epidemiológica do gênero Fannia para a dermatobiose no Brasil, como mostram os trabalhos de Paloschi et al. (1991), Brum et al. (1996), Gomes et al. (1998), Rodríguez (1998), Silva Júnior et al. (1999) e Pinto (2001).

Fanniidae é uma pequena família de dípteros caliptrados, encontrados em todas as regiões zoogeográficas do mundo, que engloba quatro gêneros e 267 espécies conhecidas (Carvalho 1993). A maioria dos indivíduos adultos desta família é encontrada em ambientes florestais. Os machos desta família apresentam o comportamento de ficar pairando no ar à espera de alimento, enquanto que as fêmeas, em geral, são encontradas na vegetação e em detritos orgânicos presentes no solo. Para Fannia pusio os adultos apresentaram uma longevidade média, em laboratório, de $26,18,5$ e 12 dias, nas temperaturas $20^{\circ} \mathrm{C}, 27^{\circ} \mathrm{C}$ e $33^{\circ} \mathrm{C}$, respectivamente (Marchiori \& Prado 1999).

Devido às evidências da importância da família Fanniidae na veiculação de ovos e, por conseguinte, na epidemiologia de $D$. hominis no Brasil, realizou-se o presente trabalho para a identificação das espécies desta família existentes na região de Campo Grande, MS; para o conhecimento das espécies mais abundantes, bem como, qual ou quais espécies estão sendo utilizadas por $D$. hominis na veiculação de seus ovos.

\section{MATERIAL E MÉTODOS}

O presente trabalho foi desenvolvido na área física do Centro Nacional de Pesquisa de Gado de Corte, da Empresa Brasileira de Pesquisa Agropecuária (Embrapa Gado de Corte), em Campo Grande, MS ( $20^{\circ} 27^{\prime} \mathrm{S}, 5^{\circ} 37^{\prime} \mathrm{W}$ e $530 \mathrm{~m}$ de altitude). Esta área, segundo a classificação de Köppen, situa-se na faixa de transição entre o clima AW tropical úmido, com estação chuvosa no verão e seca no inverno (Ometto 1981). A temperatura média do mês mais quente é superior a $22^{\circ} \mathrm{C}$, apresentando no mês mais seco, precipitação superior a 30 $\mathrm{mm}$. Os dados climáticos referentes ao período de coleta de dados no campo, que foi de 9/8/1999 a 3/8/2000, foram obtidos pela Estação Meteorológica Principal da Embrapa Gado de Corte.

Para a captura das moscas foram utilizadas cinco armadilhas, com metodologia modificada daquela empregada por Ferreira (1978), que utilizou latas pretas medindo $11 \mathrm{~cm}$ de diâmetro por $12 \mathrm{~cm}$ de altura, com oito aberturas laterais e iscas diversas (peixe, figado de galinha e fezes humanas). $\mathrm{O}$ número de aberturas foi reduzido para seis; 0 tamanho das "latas" foi aumentado para galões (capacidade para 3,6 litros), pintados originalmente com cor azul-claro; a isca utilizada foi de fígado bovino cru deteriorado, no volume de 300-350 gramas por armadilha, acondicionada em recipiente plástico de $500 \mathrm{~mL}$ coberto com tela de náilon. As aberturas laterais mediam $5 \mathrm{~cm}$ de lado e eram dispostas de forma eqüidistante na altura mediana dos galões. Eram 
feitas cortando-se a lata exceto no lado superior dessas aberturas e dobrando-se a lâmina cortada para dentro (formando abas internas). Em lugar da tampa foi colocado um funil telado, tendo a abertura menor voltada para cima, e a abertura maior (diâmetro ajustado ao diâmetro interno do galão) apoiada sobre as abas resultantes das aberturas. Na parte superior dos galões foram fixados sacos plásticos transparentes medindo $28 \times 43$ centímetros, onde as moscas eram aprisionadas no momento em que abandonavam as iscas (visto que a tendência desses dípteros é de se deslocar para o local de maior presença de luz, no caso, o topo da armadilha).

As armadilhas foram instaladas a uma altura de 1,5 metros do solo, suspensas por fios de arame e distantes cerca de 150 metros entre si, na periferia de uma mata ciliar contínua, no interior de uma pastagem com predominância da gramínea Brachiaria decumbens e presença constante de bovinos. Para evitar que formigas tivessem acesso às armadilhas, cuja possibilidade única seria pelos fios de arame, foram colocadas barreiras em todas as armadilhas, untandose com graxa de petróleo parte do galho em que as armadilhas foram suspensas (raio de $15 \mathrm{~cm}$ de extensão).

As coletas foram feitas semanalmente, sendo as iscas parcialmente renovadas a cada 30 dias. A renovação consistiu na substituição de um terço da isca, colocando-se mais fígado bovino e quantidade suficiente de água para mantê-la hidratada, de modo que o volume da isca fosse mantido constante em $2 / 3$ da capacidade do recipiente.

Após a captura, as moscas coletadas eram mortas com inseticida aerossol e levadas ao laboratório para a triagem inicial visando à separação dos indivíduos pertencentes à família de interesse neste estudo. Todos os exemplares de Fanniidae coletados foram colocados em estufa por dois dias e, em seguida, acondicionados, a seco, em frascos contendo pastilha de formol para posterior complementação da identificação. Os demais dípteros obtidos foram igualmente secos e devidamente acondicionados com identificação apropriada para serem objeto de estudos complementares.

A identificação preliminar (morfo espécies) dos Fanniidae capturados foi feita com o auxílio de chave dicotômica e a utilização de microscópio estereoscópico, no Laboratório de Sanidade Animal da Embrapa Gado de Corte, sendo posteriormente confirmada e/ou complementada pelo Prof. Dr. Cláudio José Barros de Carvalho, especialista em sistemática desta família, da Universidade Federal do Paraná, com base em exemplares de cada morfo espécie obtida, que lhe foram enviadas depois de fixadas em alfinetes entomológicos.

\section{RESULTADOS E DISCUSSÃO}

Nas capturas realizadas foram considerados apenas os dípteros pertencentes às famílias Calliphoridae, Muscidae, Sarcophagidae, Mesembrinellidae e Fanniidae, tendo sido capturados, ao todo, 547.025 espécimens. Destes, 40.629 exemplares $(7,43 \%)$ foram Fanniidae. Sugere-se que o percentual de Fanniidae aqui obtido diferiu daquele observado por Gomes et al. (1998), que foi de $14,41 \%$, em razão de que estes últimos autores empregaram armadilhas Magoon em suas capturas. Estas armadilhas utilizam-se de bezerro como isca, e os dípteros fanídeos, devido ao seu hábito zoófilo (Bates 1943), tendem a ser atraídos de modo mais eficiente do que com isca de fígado deteriorado. Dentre os quatro gêneros conhecidos, da família Fanniidae (Carvalho 1993), Fannia foi o único observado, tendo sido constatada a ocorrência de quatro espécies identificadas durante a realização desse trabalho e uma que ainda se encontra em processo de identificação (Quadro 1).
Quadro 1. Espécies de Fannia capturadas no período de ago/1999 a $\mathrm{jul} / 2000$, por meio de armadilhas iscadas com fígado bovino deteriorado, em Campo Grande, MS

\begin{tabular}{lccccc}
\hline \multicolumn{1}{c}{ Espécies } & $\begin{array}{c}\text { Fêmeas } \\
\text { (F) }\end{array}$ & $\begin{array}{c}\text { Machos } \\
(\mathrm{M})\end{array}$ & $\mathrm{F}+\mathrm{M}$ & $\begin{array}{c}\text { Total } \\
(\%)\end{array}$ & $\begin{array}{c}\text { Fêmeas } \\
(\%)\end{array}$ \\
\hline Fannia pusio & 21.906 & 3.771 & 25.677 & 63,20 & 85,31 \\
Fannia heydenii & 10.317 & 1.393 & 11.710 & 28,83 & 88,10 \\
Fannia bahiensis & 2.401 & 686 & 3.087 & 7,60 & 77,78 \\
Fannia longipila & 109 & 36 & 145 & 0,35 & 75,17 \\
Fannia sp & 6 & 4 & 10 & 0,02 & 60,00 \\
\multicolumn{1}{c}{ Total } & 34.739 & 5.890 & 40.629 & 100,00 &
\end{tabular}

De acordo com o Quadro 1, a espécie que apresentou maior abundância relativa foi $F$. pusio, representando aproximadamente dois terços do total capturado, seguida por Fannia heydenii, que participou com pouco menos de um terço e Fannia bahiensis, com pouco menos de um décimo dos indivíduos capturados. Fannia longipila e Fannia sp em conjunto responderam por apenas $0,37 \%$ do total capturado.

O percentual de fêmeas capturadas (Quadro 1) foi superior ao de machos em todas as espécies obtidas, sendo mais expressiva nas quatro espécies que apresentaram maior número de exemplares, nas quais essa relação (razão de sexo $=$ $\mathrm{F} / \mathrm{M}+\mathrm{F}$ ) foi igual ou superior a 0,75 .

Dentre as espécies da família Fanniidae capturadas, apenas $F$. heydenii foi encontrada portando ovos de $D$. hominis. A veiculação de ovos de $D$. hominis observada nesta espécie restringiu-se apenas a fêmeas, num total de 45 exemplares, o que corresponde a $0,44 \%$ do total de fêmeas coletadas. Esses resultados, no que se refere à proporção de fêmeas portando ovos de $D$. hominis, são semelhantes ao registrado por Paloschi et al. (1991) que, de um total de 54 espécimens encontrados veiculando ovos, apenas um era macho. Estes autores verificaram, também, que $F$. heydenii foi o principal vetor de ovos de $D$. hominis na região do Planalto Catarinense, representando $51,9 \%$ do total de exemplares de dípteros portando ovos. Foi, também, com respeito à família Fanniidae, a única espécie capturada veiculando ovos de $D$. hominis.

Rodríguez (1998), em Pedro Leopoldo, MG, trabalhando em diferentes ambientes, verificou que a família Fanniidae respondeu por dois terços dos espécimens vetores capturados, pertencendo os demais à Muscidae. Verificou ainda que, da família Fanniidae, apenas uma espécie, F. heydenii, atuou como vetor, sendo que, nas agrobiocenoses pastoril e eubiocenose (ambiente original), foi a única espécie de díptero capturada veiculando ovos de $D$. hominis. No ambiente estabular, que constitui uma área relativamente restrita, esta espécie representou $17 \%$ dos espécimens vetores capturados, sendo ali superada por Musca domestica (35\%) e Stomoxys calcitrans (20\%). Por isso, tanto os dados do presente trabalho, quanto aqueles registrados pelos autores acima, reforçam a importância epidemiológica de $F$ heydenii na veiculação de ovos de $D$. hominis, especialmente nas agrobiocenoses pastoril e eubiocenose.

Sugere-se que o hábito zoófilo e o tamanho moderado [5,0-5,5 mm, segundo Albuquerque et al. (1981)] de F. heydenii, 
conforme critérios estabelecidos por Bates (1943), caracterizam-na como um bom vetor de ovos de $D$. hominis. Quanto ao período de vôo diurno e atividade moderada dessa espécie, não foram feitas observações para esses comportamentos e, na literatura, não há referências sobre tais características. Entretanto, é comum encontrar representantes desta família pairando longamente no ar, em alturas variáveis (em geral, 1-3 m), segundo Carvalho (1993), especialmente no interior de domicílios e/ou clareiras na mata, onde houver incidência de radiação solar.

O número médio de ovos de $D$. hominis por exemplar de F. heydenii capturado foi de 15,98, com amplitude de quatro a 29, apresentando um desvio padrão de 7,13 e um coeficiente de variação de $44,62 \%$. Este valor está bem próximo da média de 16,3 ovos encontrada por Brum et al. (1996) em exemplares de Fannia punctipennis no município de Pelotas, RS, e da média de 16,4 ovos por exemplar de Fannia sp, registrada por Gomes et al. (1998), em Mato Grosso do Sul. É, entretanto, inferior àqueles verificados por Paloschi et al. (1991), que registraram uma média de 21,6 ovos (amplitude de 10 a 55) e por Rodríguez (1998), que obteve 23,98 (amplitude de 7-63).

Só foram constatadas posturas na região abdominal do vetor e a posição dos ovos variou entre indivíduos, sendo sempre laterais. As posturas foram assim distribuídas: lateral direita $(15,6 \%)$, lateral esquerda $(24,4 \%)$, látero-ventral direita $(24,4 \%)$, látero-ventral esquerda $(33,3 \%)$ e látero-dorsal direita $(2,3 \%)$. Agrupando essas posições em apenas direita e esquerda, para efeito de comparação com os relatos de outros autores, resultam, respectivamente, $42,3 \%$ e $57,5 \%$. Evidencia-se, portanto, uma preferência de $D$. hominis por ovopositar na região lateral esquerda do abdome de $F$. heydenii. Essa preferência foi anteriormente verificada por Paloschi et al. (1991), e representou $80,3 \%$ dos exemplares de $F$. heydenii capturados, já incluídos os 10,3\% de indivíduos nos quais as posturas encontravam-se aderidas ao tórax (exclusivamente no lado esquerdo). Rodríguez (1998), nesta mesma espécie, encontrou todas as posturas na lateral esquerda dos indivíduos capturados nos ambientes estabular e pastoril, sendo que nos espécimens capturados na eubiocenose, $71,3 \%$ das posturas localizavam-se no lado esquerdo.

A dinâmica populacional de Fannia heydenii em comparação com os dados climáticos de temperatura média e precipitação total mensais, estão representados na Fig. 1. Maiores níveis populacionais ocorreram entre agosto e novembro de 1999 e maio/junho de 2000, com o principal pico ocorrendo em agosto de 1999 e um segundo pico, de menor intensidade, em junho de 2000 . O período acima, apesar de incluir os meses de menor precipitação do ano (Fig. 1), apresentou temperaturas amenas, favorecendo o desenvolvimento desta espécie, que se mostrou mais numerosa na faixa de temperatura entre $15^{\circ} \mathrm{C}$ e $20^{\circ} \mathrm{C}$ (Fig. 1). Tal hipótese é aqui proposta diante do fato de Paloschi et al. (1991) terem capturado maior número desta espécie nos meses de verão e outono, visto que o clima naquela região é classificado como mesotérmico, úmido e com verão fresco, segundo a classificação de Köppen, citado por Ometto (1981), com temperaturas médias próxi-

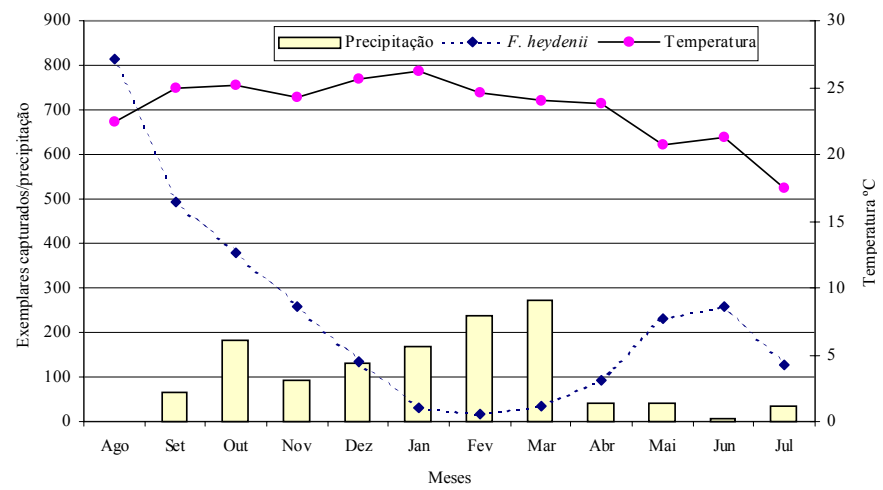

Fig. 1. Valores mensais da temperatura média, da precipitação total, e do número médio de exemplares por coleta, de Fannia heydenii capturados no período de 09/08/1999 a 03/08/2000, por meio de armadilhas com iscas de fígado bovino deteriorado, em Campo Grande, MS.

mas àquelas aqui verificadas durante o período de maior ocorrência de $F$. heydenii.

O mês em que se verificou maior número de fêmeas portando ovos de $D$. hominis foi agosto de 1999 , com 25 exemplares, seguido do mês de setembro com oito exemplares. Estes resultados confirmam aqueles encontrados por Gomes et al. $(1988,1996)$ ao verificarem que, apesar das infestações pelo berne ocorrerem durante $o$ ano todo, as maiores infestações foram observadas no período compreendido entre os meses de agosto a outubro e durante o mês de dezembro, sendo o maior pico em outubro. Visto que a duração aproximada do período larval de $D$. hominis em bovinos é de 40 a 60 dias (Jobsen \& Mourier 1972), o maior número de moscas portando ovos de $D$. hominis no período de agosto e setembro, na região deste estudo, tem relação com as infestações do berne em bovinos, principalmente, nos meses de setembro e outubro. A distribuição sazonal de larvas de D. hominis, no município de Governador Valadares, MG, apontou que a infestação dos animais foi maior entre os meses de outubro e dezembro, que compreende a parte inicial do período chuvoso (Maia \& Guimarães 1985). Resultados semelhantes foram também obtidos por Brito \& Moya Borja (2000), na região de Piraí, RJ.

Contudo, levando-se em conta que a metodologia aqui empregada não cobre a diversidade de espécies incriminadas na veiculação de ovos de $D$. hominis, supõe-se que as infestações por berne localmente verificadas a partir de novembro podem estar associadas a uma ou mais destas espécies.

\section{REFERÊNCIAS}

Albuquerque D.O., Pamplona D. \& Carvalho C.J.B. 1981. Contribuição ao conhecimento dos Fannia R. D., 1830 da região neotropical (Diptera, Fanniidae). Arq. Mus. Nac., Rio de Janeiro, 56 (1): 9-34.

Bates M. 1943. Mosquitos as vectors of Dermatobia in Eastern Colombia. Ann. Ent. Soc. Amer. 36 (1): 21-24.

Brito L.G. \& Moya Borja G.E. 2000. Flutuação sazonal de Dermatobia hominis em peles bovinas oriundas de matadouro. Pesq. Vet. Bras. 20 (4): 151-154.

Brum J.G.W., Gentilini F., Pinto L.S. \& Vianna E.E.S. 1996. Fannia punctipennis 
(Diptera: Fanniidae) vetor de ovos da Dermatobia hominis (Oestridae: Cuterebrinae) em Pelotas, RS. Revta Bras. Parasitol. Vet. 5: 59-60.

Carvalho C.J.B. 1993. A catalogue of the Fanniidae and Muscidae (Diptera) of the Neotropical region. Sociedade Brasileira de Entomologia, São Paulo. 201p.

Ferreira M.J.M. 1978. Sinantropia de dípteros muscóideos de Curitiba, Paraná. I: Calliphoridae. Revta Bras. Biol. 38 (2): 445-454.

Gomes A., Sousa J.C., Resende A.M. \& Curvo J.B.E. 1988. Distribuição corporal e sazonalidade do berne (larva de Dermatobia hominis) em bovinos tratados ou não com flor de enxofre. Pesq. Agropec. Bras. 23 (8): 825-829.

Gomes A., Honer M.R. \& Silva R.L. 1996. Intensidade parasitária de larvas de Dermatobia hominis (L. Jr., 1781) (Diptera: Cuterebridae) em bovinos de diferentes raças cruzadas extensivamente na região de Cerrado em Mato Grosso do Sul. Revta Bras. Parasitol. Vet. 5 (2): 103-106.

Gomes A., Honer M.R., Koller W.W. \& Silva R.L. 1998. Vetores de ovos de Dermatobia hominis (L. Jr., 1781) (Diptera: Cuterebridae) na região de Cerrados do Mato Grosso do Sul, Brasil. Revta Bras. Parasitol. Vet. 7 (1): 37-40.

Grisi L., Massard C.L., Moya Borja G.E. \& Pereira J.B. 2002. Impacto econômico das principais ectoparasitoses em bovinos no Brasil. Hora Vet. 21 (125): 8-10.

Guimarães J.H. \& Papavero N.A. 1999. Myiasis in man and animals in the Neotropical region; bibliographic database. Plêiade/FAPESP, São Paulo. 308p.

Jobsen J.A. \& Mourier H. 1972. The morphology of the larval instars and pupa of Dermatobia hominis L. Jr. (Diptera: Cuterebridae). Entomol. Berich. 32: 218-224.

Maia A.A.M. \& Guimarães M.P. 1985. Distribuição sazonal de larvas de Dermatobia hominis (Linnaeus Jr., 1781) (Diptera: Cuterebridae) em bovinos de corte da região de Governador Valadares - Minas Gerais. Arq. Bras. Med. Vet. Zootec. 37 (5): 469-475.

Maia A.A.M. \& Gomes A.G. 1988. Vetores de Dermatobia hominis, Linnaeus Jr., 1781 (Diptera: Cuterebridae) na região de Uberaba, Minas Gerais. Revta Fac. Med. Vet. Zootec. USP 25 (1): 47-51.
Marchiori C.H. \& Prado A.P. 1999. Tabelas de vida de Fannia pusio (Wied.) (Diptera: Fanniidae). An. Soc. Entomol. Bras. 28 (3): 557-563.

Mateus G. 1967. El nuche y su ciclo de vida. Revta Inst. Colomb. Agropec. 2 (1): 3-19.

Mateus V.G. 1979. Dermatobiosis. Anais do I Seminário Nacional sobre Parasitoses dos Bovinos, Campo Grande, MS, p. 315-329 e 331-333.

Oliveira G.P. 1986. Distribuição sazonal de dípteros muscóides sinantrópicos, simbovinos e foréticos de Dermatobia hominis L. Jr., em São Carlos, Estado de São Paulo. I. Estábulo. Arqs Biol. Tecnol. 29: 311-325.

Ometto J.C. 1981. Bioclimatologia vegetal. Agronômica Ceres, São Paulo. 425p.

Paloschi C.G., Ramos C.I., Souza A.P., Bellato V., Sartor A.A. \& Dalagnol C.A. 1991. Vetores de ovos de Dermatobia hominis no Planalto Catarinense. Pesq. Agropec. Bras. 26 (11/12): 1872-1883.

Pinto S.B. 2001. Aspectos bioecológicos e imunológicos de Dermatobia hominis (Linnaeus Jr., 1781) (Diptera: Oestridae). Dissertação, Universidade Federal do Paraná, Curitiba. 135p.

Rodríguez Z.B. 1998. Dermatobia hominis (L. Jr., 1781) (Diptera: Oestridae: Cuterebrinae): ciclo silvestre e ecologia das infestações de bovinos pelo berne no município de Pedro Leopoldo, MG, Brasil. Dissertação, Universidade Federal de Minas Gerais, Belo Horizonte. 101p.

Roncalli R.A. 1984. The biology and the control of Dermatobia hominis, the tropical warble fly of Latin America. Prev. Vet. Med. 2: 569-578

Sancho E., Caballero M. \& Ruíz-Martinez I. 1996. The associated microflora to the larvae of human bot fly Dermatobia hominis L. Jr. (Diptera: Cuterebridae) and its furuncular lesions in cattle. Mem. Inst. Oswaldo Cruz 91: 293-298.

Schonhorst E.O. 1988. Revisão bibliográfica sobre a larva de Dermatobia hominis: o berne. Hora Vet. 7 (41):47-50.

Silva Júnior V.P. da, Leandro A. de S. \& Borja G.E.M. 1999. Descrição inédita de Fannia sp. (Diptera: Fanniidae), como vetor dos ovos de Dermatobia hominis (Diptera: Cuterebridae) em Seropédica, RJ. Revta Bras. Clín. Vet. 6(3): 173-174. 